\title{
IAS15921 AGR-5/6/7 LEUCO Kernel Fabrication Readiness Review
}

Project\# (s) 23841, 29412

The INL is a

U.S. Department of Energy

National Laboratory

operated by

Battelle Energy Alliance

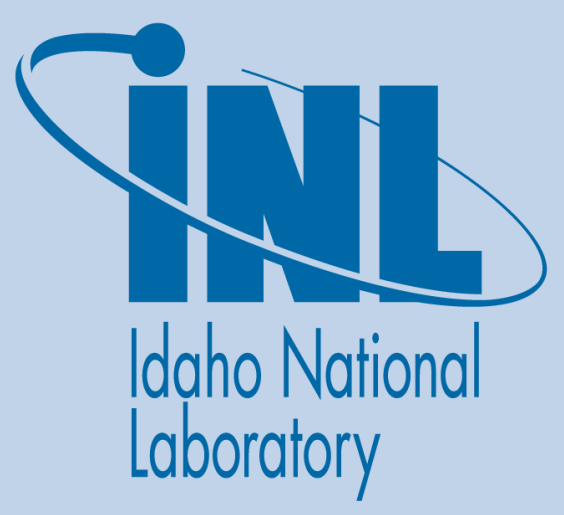

D. W. Marshall

K. W. Bailey

February 2015

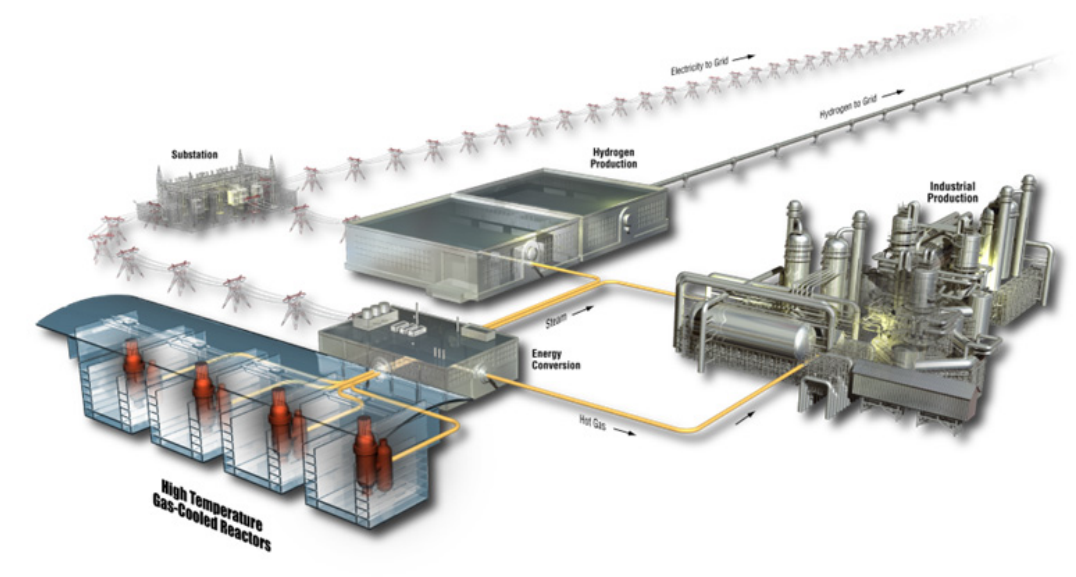




\section{DISCLAIMER}

This information was prepared as an account of work sponsored by an agency of the U.S. Government. Neither the U.S. Government nor any agency thereof, nor any of their employees, makes any warranty, expressed or implied, or assumes any legal liability or responsibility for the accuracy, completeness, or usefulness, of any information, apparatus, product, or process disclosed, or represents that its use would not infringe privately owned rights. References herein to any specific commercial product, process, or service by trade name, trade mark, manufacturer, or otherwise, does not necessarily constitute or imply its endorsement, recommendation, or favoring by the U.S. Government or any agency thereof. The views and opinions of authors expressed herein do not necessarily state or reflect those of the U.S. Government or any agency thereof. 


\title{
IAS15921 AGR-5/6/7 LEUCO Kernel Fabrication Readiness Review
}

\author{
D. W. Marshall \\ K. W. Bailey
}

February 2015

Idaho National Laboratory

INL ART TDO Program

Idaho Falls, Idaho 83415

http://www.inl.gov

Prepared for the

U.S. Department of Energy

Office of Nuclear Energy

Under DOE Idaho Operations Office

Contract DE-AC07-05ID14517 

ART Program

\section{IAS15921 \\ AGR-5/6/7 LEUCO Kernel Fabrication Readiness \\ Review}

INL/EXT-15-34306

February 2015

Prepared by:
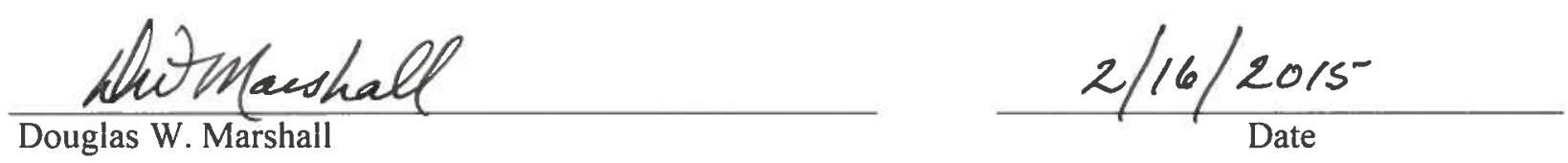

Fuel Design and Development

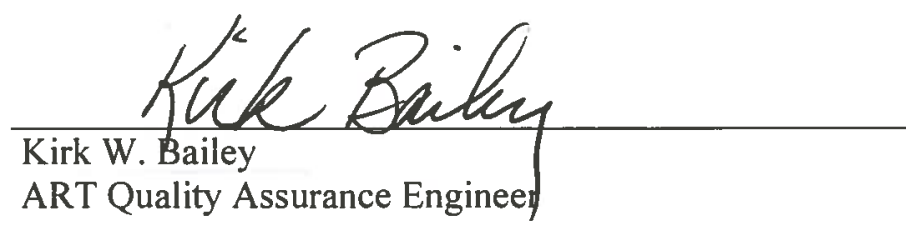

$\frac{2 \cdot 16 \cdot 2015}{\text { Date }}$

Approved by:
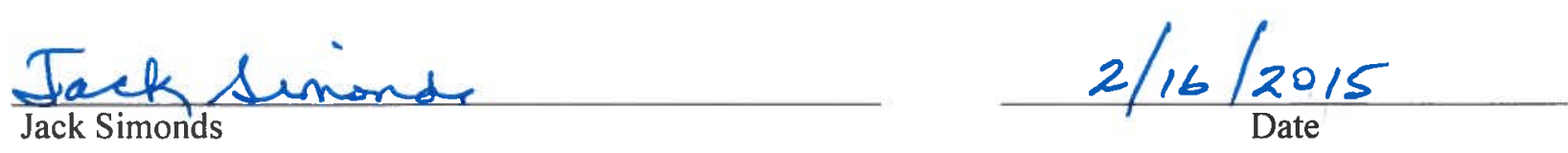

AGR Fuel Development and Qualification Project

Manager

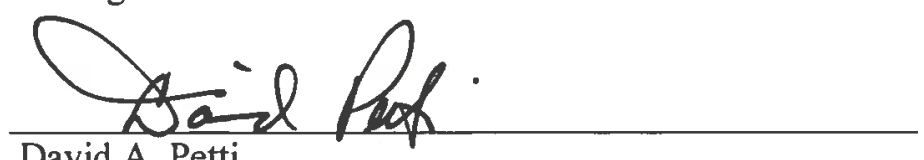

David A. Petti

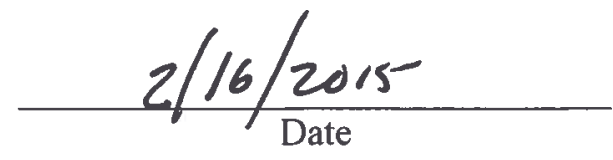

ART Co-National Technical Director 


\section{SUMMARY}

A readiness review of the kernel fabrication line at Babcock and Wilcox Nuclear Operations Group of Lynchburg, Virginia took place January 13 through the $15^{\text {th }}$ of 2015 to establish their readiness status to produce low-enriched uranium carbide/oxide fuel kernels for INL in support of Advanced Gas Reactor$5 / 6 / 7$ experiment project plans. The review team was able to evaluate all pertinent areas and determined that B\&W NOG-L's kernel fabrication line was conditionally ready to produce fuel kernels. Seven issues were identified that require resolution prior to kernel line start-up. Three additional issues were identified requiring resolution, but which are not a prerequisite to start-up of the line. Of the seven prerequisite issues, three have been resolved during the preparation of this report.

The prerequisite issues are:

1. IAS15921 RI001: 2.5-inch kernel sintering furnace (needed for kernel fissure reduction studies) is out-of-service pending verification of the Facility Alarm System function and Items Relied upon For Safety (IROFS). B\&W NOG-L self-identified.

2. IAS15921 RI002: 6-inch kernel sintering furnace (needed for production) is out-of-service. B\&W NOG-L self-identified.

3. IAS15921 RI003: Nuclear criticality safety evaluation for the kernel gel wash and dry stations needs to be updated to reflect present equipment configuration. B\&W NOG-L self-identified.

4. IAS15921 RI004: Correspondence between measured pump rates and uncalibrated flow meter readings, used to verify that the set flow rate has not drifted during kernel production, is not logged for operator reference. INL identified.

5. IAS15921 RI005: Ultra-high purity carbon monoxide gas cylinders had certificate of analysis with an indicated retest date for some, but not all cylinders. Retest dates had lapsed. Meaning of the retest dates was unclear. INL identified. Resolved.

6. IAS15921 RI006: The chemical inventory was not correlated with the chemical quantities needed to start and to complete kernel fabrication for all chemicals used in the process. INL identified. Resolved.

7. IAS15921 RI007: Training records presented as evidence lacked line-manager signature and date and evidence that the training had been finalized. INL identified. Resolved. 


\section{ACKNOWLEDGEMENTS}

Personnel at the Babcock and Wilcox Nuclear Operations Group Lynchburg (B\&W NOG-L) facility were very accommodating to the review team and made a concerted effort to obtain and provide the requested data and/or information in a timely manner. The B\&W NOG-L team welcomed the review and took the position that external readiness reviews lead to improvements in their performances. The friendly and cooperative interaction with the Idaho National Laboratory (INL) review team was appreciated. 


\section{CONTENTS}

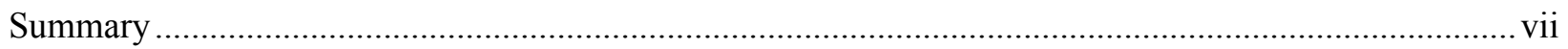

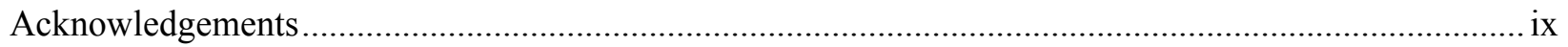

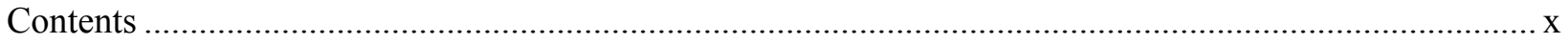

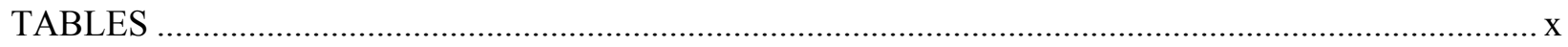

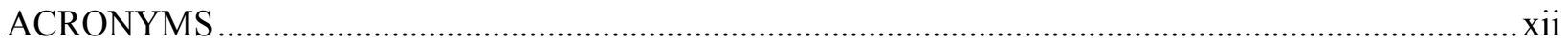

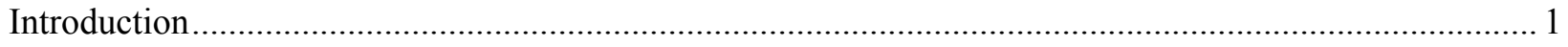

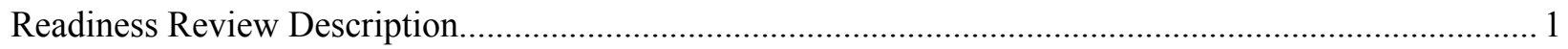

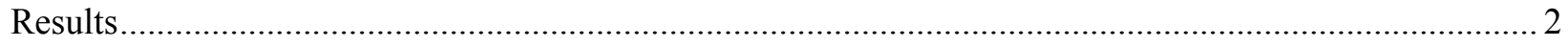

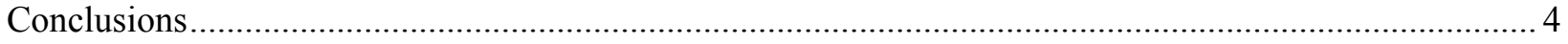

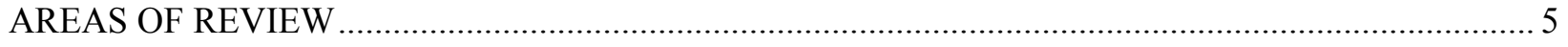

Process Instrumentation and Measuring \& Test Equipment (M\&TE) Calibrations .......................... 5

Approval, Control, and Accessibility of Operation Procedures .................................................... 6

Process Record Control and Accessibility............................................................................ 7

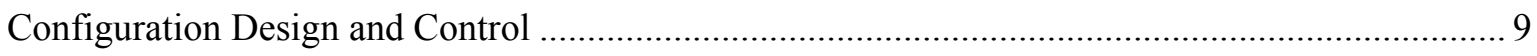

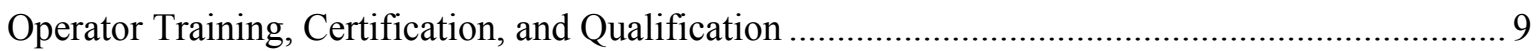

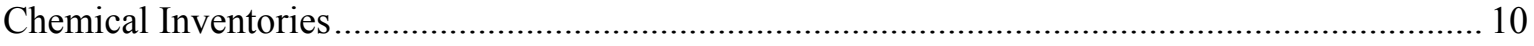

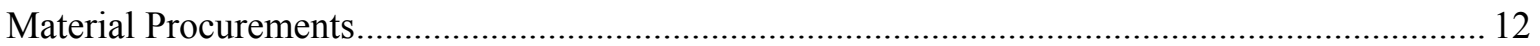

Operability of Essential Kernel Fabrication Equipment............................................................ 12

Corrective Action System Effectiveness to Capture "Lessons Learned" ........................................ 13

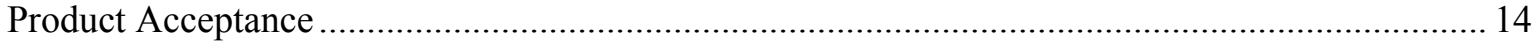

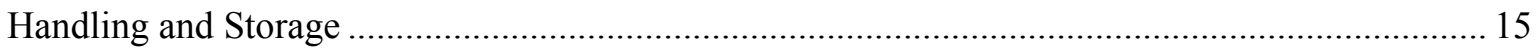

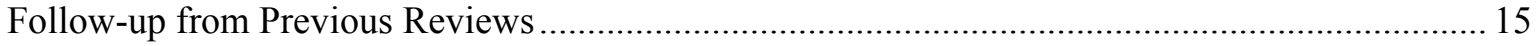

Issues 17

Appendix 1 Readiness Review Criteria AGR-5/6/7 LEUCO Kernel Fabrication ..................................... 1

\section{TABLES}

Table 1 Objective Evidence for Process Instrumentation and M\&TE................................................ 6

Table 2. Objective evidence reviewed for document control. ................................................................. 6

Table 3. Objective evidence for record control and accessibility........................................................ 8

Table 4. Objective evidence for configuration control of equipment and processes. ............................... 9

Table 5. Objective evidence for operator training, certification, and qualification. ................................ 10

Table 6. Objective evidence for chemical inventory and suitability .................................................... 11 
Table 7. Objective Evidence for Essential Kernel fabrication Equipment

Table 8. Objective Evidence for Product Acceptance

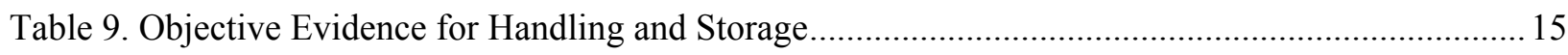

Table 10. Objective Evidence for satisfactory resolution of concerns from a previous review. 


\section{ACRONYMS}

ADUN

AGR

ART

ASME

B\&W NOG-L

BEA

$\mathrm{CO}$

CR

FAS

HEU

INL

IROFS

LEUCO

M\&TE

NCS

NQA-1

NU

PNNL

PVSS

$\mathrm{UCO}$

UHP acid-deficient uranyl nitrate solution

Advanced Gas Reactor

Advanced Reactor Technology

American Society of Materials Engineers

Babcock \& Wilcox Nuclear Operations Group - Lynchburg

Battelle Energy Alliance, LLC

carbon monoxide

change request

facility alarm system

highly-enriched uranium

Idaho National Laboratory

items relied on for safety

low-enriched uranium carbide/oxide (kernels)

measuring and test equipment

nuclear criticality safety

(ASME) Nuclear Quality Assurance Standard

natural uranium

Pacific Northwest National Laboratory

process variable specification sheet

uranium carbide/oxide

ultra high purity 


\section{INTRODUCTION}

In preparation for forming low-enriched uranium carbide/oxide (LEUCO) fuel kernels for the Advanced Gas Reactor (AGR) fuel development and qualification program, Idaho National Laboratory (INL) conducted an operational readiness review of the Babcock \& Wilcox Nuclear Operations Group Lynchburg (B\&W NOG-L) procedures, processes, and equipment from January 13 - January 15, 2015.

The readiness review focused on quality assurance requirements taken from the American Society Mechanical Engineers (ASME) Nuclear Quality Assurance Standard (NQA-1-2008, 1a-2009), preparedness to restart fuel kernel fabrication processes, response to a recent occurrence at the $\mathrm{B} \& \mathrm{~W}$ NOG-L facility related to preparation of acid-deficient uranyl nitrate solution (ADUN), and a relook at concerns noted in a previous review.

Topical areas open for the review were communicated to B\&W NOG-L in advance of the on-site visit to facilitate the collection of objective evidence attesting to the state of readiness. The topical areas are listed in Appendix 1.

\section{READINESS REVIEW DESCRIPTION}

A readiness review was held at the facilities of Babcock and Wilcox Nuclear Operations Group of Lynchburg, Virginia, an NQA-1-2008/1a-2009 INL qualified supplier of nuclear fuels research and development services and fuel provider. The purpose of the readiness review was to evaluate and determine readiness status of their uranium carbide/oxide (UCO) kernel fabrication line.

The opening meeting was held January 13,2015. The review took place over three days and a closing meeting was held January 15, 2015. The review was performed by Lead Auditor, Kirk Bailey and Fuels Design and Development Engineer, Doug Marshall as a Technical Specialist. The basis of the review was a checklist developed from sections of NQA-1-2008/1a-2009, "Quality Assurance Requirements for Nuclear Facility Applications" as they applied to the fabrication processes; including:

- Process Instrumentation and Measuring \& Test Equipment (M\&TE) Calibrations

- Approval, Control, and Accessibility of Operation Procedures

- Process Record Control and Accessibility

- Configuration Design and Control

- Operator Training, Certification, and Qualification

- Chemical Inventories

- Material Procurements

- Operability of Essential Kernel Fabrication Equipment

- Corrective Action System Effectiveness to Capture "Lessons Learned"

- Product Acceptance

- Handling and Storage

Details of the reviews conducted in each topical area are found in the section titled "Areas of Review". 


\section{RESULTS}

The readiness review produced issues in two categories, 1) those needing resolution prior to beginning kernel fabrication and 2) issues that must be resolved but did not require resolution prior to kernel fabrication. Notable practices were identified and included in the list of results.

Please note that some of the issues have been resolved either at the time of the audit or prior to producing this report. The list below reflects this differentiation.

\section{NOTABLE PRACTICES:}

Peer review of spreadsheet calculations and data entries for broth "recipe sheets" is a notable practice. The peer review, however, failed to identify an incorrect urea:uranium mole ratio that was propagated over multiple forming runs.

\section{ISSUES PREREQUISITE FOR LINE START UP:}

Prerequisite issues unresolved at the time of the report issuance:

- RI001 -The 21/2" kernel sintering furnace (WS-140) is out-of-service pending facility alarm system (FAS) and items relied on for safety (IROFS) review and release.

- RI002 -The 6" kernel sintering furnace (WS-145) is out-of-service and not ready for operation. The status of WS-145 does not need to be changed prior to operating either the forming columns or WS-140.

- RI003 - The kernel gel wash and dry stations are out-of-service because the nuclear criticality safety Monte Carlo model used for criticality safety calculations does not reflect the current asbuilt configuration inside the hood. Notably, this was self-identified by B\&W NOG-L and resolution was in progress before the readiness review was conducted. The reason for the discrepancy is a B\&W NOG-L concern.

- RI004 - Forming column solution pumps and their settings are determined by adjusting the pump rate and measuring the mass of pumped fluid over a time interval. The calculated flow rate and mass flow meter readings need to be compared and logged. This practice enables the uncalibrated flow meter readings to be used as relative measures for solution flow rates, thus allowing the meter readings to be used in verifying that flow rates remain in the $\pm 5 \%$ window given in the B\&W NOG-L operating procedure.

Prerequisite issues and resolved at the time of the report issuance:

- RI005 - Airgas Certificates of Batch Analysis for three ultra-high purity (UHP)-carbon monoxide (CO) gas lots were presented as objective evidence of readiness. Two of the gas batches were analyzed by a Texas laboratory in 2010 with indicated retest dates that lapsed in 2013 . The third gas batch was analyzed by a New Jersey laboratory in 2012, but did not include a retest date. Whether the UHP-CO is suitable for use is indeterminate until the meaning of the retest dates on two batches and the lack of same on the other are understood and addressed.

B\&W NOG-L consulted with Airgas regarding the discrepancy and the meaning of the retest dates. Airgas indicated that the ultra-high purity carbon monoxide gas did not have a shelf-life and that the Texas laboratory used the "retest" date to indicate the retention period on the Certificate of Batch Analysis.

- RI006 - The solid and liquid chemical inventory needs to be compared with what is needed to start and to complete kernel fabrication. Any shortfall in the inventory requires objective evidence that the needed chemicals will be acquired in time for processing (e.g., minimum inventory triggers for reordering, purchase orders, or procurement plan). 
B\&W NOG-L has completed an estimate of the chemicals needed. Two chemicals are not in sufficient supply to complete the kernel forming activity, but sufficient is on-hand to start forming. Requisitions are in process to acquire the sufficient chemical to meet fabrication needs.

- RI007 - A concern was noted when reviewing old operator training records for kernel fabrication in 2012. Training exams were presented as objective evidence that lacked signatures from the cognizant engineer and the front-line manager, dates, procedure revision numbers, and evidence that the exams were graded. This is noted as a weakness only because the operator qualifications associated with the documentation have expired and will need to be redone prior to kernel line operation.

Copies of recent operator qualification exams and training records for the kernel fabrication processes have been provided showing that the exams have been graded, the applicable procedure revision is noted, and the records have been authenticated by frontline-manager signatures and dates. Training records have been revised to include signature blocks for the manager signatures and dates.

\section{ISSUES REQUIRING RESOLUTION BUT NOT PREREQUISITE FOR START-UP.}

- UI001 - Some QA records, specifically training attendance record, Form N-50 "Employee Review of Area Documents," lack the authenticating manager signature and date as required in NQA-1 Requirement 17 300(a). This concern was noted in a previous readiness review, but has not been adequately resolved or implemented.

Copies of recent operator qualification exams and training records for the kernel fabrication processes have been provided showing that the documents have been revised to include a block for frontline-manager signatures and date to authenticate the record.

- UI002 - Instrument calibrations appear to be in order, but a weakness was noted. The nature of work performed at B\&W NOG-L does not lend itself well to the use of external agencies to certify the calibration services laboratory to nationally recognized performance standards, such as ISO 10012 or ISO 17025. A link between the B\&W NOG-L calibration services and the national performance standards could not be verified during the review and remains indeterminate.

- UI003 - No objective evidence was presented regarding the "periodic" evaluation of technician skills in the calibration services that they provide. 


\section{CONCLUSIONS}

B\&W NOG-L demonstrated a kernel fabrication line and support staff and services capable of fabricating low-enriched UCO (LEUCO) kernels for INL. This line produced kernels as recently as 2012. It has been in standby status until a need expressed by INL to produce LEUCO fuel kernels for AGR $5 / 6 / 7$. Several issues were identified that require resolution before the line will be production ready. These are identified in the results section. From this review the follow conclusions are made:

- Procedures, forms and other support documentation are in order and ready for use

- Availability of qualified personnel for operations remains an area of concern but is partially mitigated by the hiring and training processes in place as well as the depth of knowledge and experience of a tenured engineering and support staff.

- Record authentication problems persist and require attention to detail and diligence to resolve.

- The production (6") sintering furnace will be brought into a full-readiness status before it is needed for fabricating LEUCO fuel kernels for the AGR-5/6/7 experiments. Readiness of the production furnace has a lower priority than other equipment readiness because it will not be needed until several weeks after kernel fabrication commences.

- Subject to the outstanding prerequisite issues, and internal B\&W NOG-L evaluation, kernel wash/dry stations and the $2 \frac{1}{2}$ " kernel sintering furnace will be ready for commencing LEUCO kernel fabrication for the initial studies to reduce the incidence of kernel fissures. 


\section{AREAS OF REVIEW}

\section{Process Instrumentation and Measuring \& Test Equipment (M\&TE) Calibrations}

Measuring and Test equipment (M\&TE) including tools, gages, and instruments used for activities affecting quality shall be controlled, calibrated at specific periods, adjusted, and maintained to required accuracy limits.

The B\&W NOG-L calibration services group managed by the Calibration Services Manager provides for calibration services for the B\&W N OG-L group performing fuel development activities under Quality Assurance Plan OP-1003186, Quality Plan for Uranium Processing and Research Reactors (u), Rev. 9. Implementing procedures reviewed included OP-1000180, Calibration Services, Rev 24, and CSP-1015, Guide to OP-1000180 Applications, Rev. 08.

A tour of the processing areas was held. All equipment was housed behind protective doors and casements. Eight thermocouples were examined for calibration status from the $2 \frac{1}{2}$ " kernel sintering furnace and gel wash and dry stations. In all cases these sensors were found to be within stated calibration periods. During the discovery process, the auditors became aware that the Calibration Services had at one time been certified to ISO-10012. Currently, the Services were no longer certified. Three areas of questioning were posed covering procedures and procedure maintenance, employee training and records.

As mentioned before, all reviewed records were in order and current within calibration periods listed. Training for Calibration Services specialists was described as a plan where the technical leads would be trained using internal mentoring, on-the-job and classroom training and external classes. The records presented for this training were an employee who at the time of the audit was retired with many years of services with the company and a long list of training evidence and his replacement. The replacement employee training record had the new employee (new to this job) review and checklist and some training evidence however, no external training was evident. The procedures from the quality manual to implementing calibration services all appeared to be in order. Audit records from two audits showed that Calibration Services was audited to internal procedures.

A weakness was identified associated with this area for the following reasons: Training records were less-than-adequate for the employee taking over as technical lead, for him to fulfill his role as a calibration technical specialist and Lead, according to the Calibrations Services Manager he needs outside training as well as the internal mentoring and instruction he received in house. Additionally no evidence of periodic evaluation was made available. Finally, audit reports demonstrated internal auditing was taking place but the requirements used were the internal procedures. It was not clear whether or not NQA1-2008, 1a-2009 requirements were covered during these audits or another recognized standard was used as the basis for the audit source material.

This area is compliant to NQA-1 requirements and considered ready for use however, issues were identified in the review that need resolving, but are not prerequisite for kernel fabrication. They include issues UI002 and UI003.

Objective evidences reviewed for this area are given in Table 1: 
Table 1 Objective Evidence for Process Instrumentation and M\&TE

\begin{tabular}{|l|c|}
\hline \multicolumn{1}{|c|}{ Evidence Reviewed } & Revision or Date \\
\hline OP-1000180, Calibration Services & 24 \\
\hline OP-1003186, Quality Plan for Uranium Processing and Research Reactors (u) & 9 \\
\hline CSP-1015, Guide to OP-1000180 Applications & 8 \\
\hline Gage / Instrument Calibration Procedure & 19 \\
\hline CSP-1006, New Employee 90-Day Training Procedure & 4 \\
\hline Training Record for Robert T Lipscomb & $1 / 14 / 2015$ \\
\hline Training Record for Rick McNeely & $1 / 14 / 2015$ \\
\hline
\end{tabular}

\section{Approval, Control, and Accessibility of Operation Procedures}

Activities affecting quality must be prescribed by and performed in accordance with documented instructions or procedures. Such documents are to be controlled to ensure that changes are reviewed, approved, and authorized for use prior to implementation in the field and only the latest revisions are available to the performers. Process requirements and acceptance criteria are to be based on documented specifications.

The B\&W NOG-L procedures and instructions are controlled by a change request generated in accordance with their document control procedure (Quality Work Instruction QWI 5.1.12, "Change Management"). Proposed changes to a procedure/instruction are reviewed by subject matter experts and management to ensure that no unforeseen adverse consequences may be created by the change. Once concurrences and unit manager approval are obtained, the revision is incorporated electronically into the procedure/instruction and the electronic document is uploaded onto a plant-wide computer system known as Solumina. Operating personnel access the procedure/instruction via the Solumina system, thus ensuring that they are performing work to the most recent revision.

One exception to the use of Solumina involves batch blending instructions, which are delivered to the operating personnel on a hard copy instruction sheet with signatures of two people from the engineering department.

The approval, control, and accessibility of the operating procedures and instructions were found to be effective and compliant with NQA-1 requirements. Documents reviewed to assess the effectiveness of the B\&W NOG-L document control practices are referenced in Table 2.

Table 2. Objective evidence reviewed for document control.

\begin{tabular}{|l|c|}
\hline \multicolumn{1}{|c|}{ Evidence Reviewed } & Revision or Date \\
\hline “LEU Liquid Blend Run Sheet” (J52L-16-02423) & $9 / 20 / 12$ \\
\hline "Process Variable Specification Sheet for ADUN Blending” (J52L-16-02423) & $9 / 17 / 12$ \\
\hline Change Request (CR), "Revise OP-1014625 to Add Steam Condensate Clearing (U)" & $10 / 14 / 12$ \\
\hline OP-1014625, “Operating Procedure For Broth Preparation, Particle & 10 \\
\hline
\end{tabular}




\begin{tabular}{|l|c|}
\hline \multicolumn{1}{|c|}{ Evidence Reviewed } & Revision or Date \\
\hline Formulation/Aging For Advanced Gas Reactor Program" & 4 \\
\hline $\begin{array}{l}\text { OP-1022961, "Sintering for AGR and Other SFF Contracts in the 2.5 Inch Furnace at } \\
\text { WS140" }\end{array}$ & NA \\
\hline PPO-0920016-002, "2.5-Inch Fluid Bed Sintering for Advanced Gas Reactor" & $12 / 18 / 12$ \\
\hline $\begin{array}{l}\text { Process Variable Specification Sheet (PVSS) screen shot } \\
\text { PPO no. (tracking): 000001107394 } \\
\text { Parent serial no.: J52L-16-59516 }\end{array}$ & NA \\
\hline $\begin{array}{l}\text { PVSS “AGR Fluid Bed Sintering in Centorr Furnace (21/2”")" } \\
\text { (Batch: G73AB-NU-59116) }\end{array}$ & NA \\
\hline $\begin{array}{l}\text { PVSS Form CRF-0211, "Forming/Carbon Dispersion/Wash/Dry for AGR Process } \\
\text { Variable Update" (Batch: J52L-16-29129) }\end{array}$ & NA \\
\hline $\begin{array}{l}\text { PVSS Form CRF-0212, "Forming/Carbon Dispersion/Wash/Dry Data for Advanced } \\
\text { Gas Reactor" (Batch: J52L-16-29129) }\end{array}$ & $1 / 24 / 13$ \\
\hline $\begin{array}{l}\text { PVSS screen shot } \\
\text { PPO no. (tracking): 000001105253 } \\
\text { Parent serial no.: J52L-16-29128 }\end{array}$ & \\
\hline $\begin{array}{l}\text { PVSS screen shot } \\
\text { PPO no. (tracking): 000001105247 } \\
\text { Parent serial no.: J52L-16-29122 }\end{array}$ & $12 / 5 / 12$ \\
\hline $\begin{array}{l}\text { Quality Work Instruction 5.1.12, "Change Management” } \\
\text { Revision History Log, "Revise OP-1014625 to Add Steam Condensate Clearing } \\
\text { (U)," Change Review Minutes }\end{array}$ & $10 / 8 / 12-$ \\
\hline
\end{tabular}

\section{Process Record Control and Accessibility}

Requirements for process record control and accessibility applicable to AGR LEUCO kernel fabrication are that records are validated or authenticated by authorized personnel. The expectation is that records will have the signature and date of the authorized personnel before the record goes into storage.

Records presented to the review team were generally compliant and, in some instances, exceeded the requirement (e.g., work instructions for batch blending operations were issued under signature of two authorized engineers; providing peer review of the instruction). However, training records (presented as objective evidence) with lists of operations personnel that had completed training were not signed and dated by a manager. This non-compliant issue had been addressed in a previous visit of 2014 and the resolution was not fully implemented at the time of review. Written exams used for operator qualification were presented as objective evidence of B\&W NOG-L practices from qualifications on the kernel forming processes in 2012. Within this group of exams, three were for a single operator and all three were taken on the same day. None of the three had an authorizing signature by the frontline manager and one exam showed no evidence of having been graded, did not indicate the revision to the operating procedure for which is was applicable, and was missing the signature and date of the engineer grading the exam.

Had the nonconformities, noted above, been for current qualification or requalification of the operating personnel, the review team would have deemed B\&W NOG-L as not ready for these criteria. More current qualification exams could not be presented to the review team for examination, at the time of the review, because the exams have been revised and needed review for possible confidential and business sensitive information and external release. Subsequent to the review and during preparation of this report, B\&W NOG-L provided copies of recent qualification exams for two operators and a blank 
training form, N-50 "Employee Review of Area Documents," Revision 5. Both exams were graded, signed and dated by the grading engineer, and signed and dated by a manager to authenticate the records. The N-50 form revision incorporates a signature block for the authenticating manager's signature and date.

Process record control and accessibility are considered to be compliant with NQA-1 requirements. The issue identified during the review (UI001) has been resolved. Objective evidences reviewed are given in Table 3.

Table 3. Objective evidence for record control and accessibility.

\begin{tabular}{|c|c|}
\hline Evidence Reviewed & Revision or Date \\
\hline Change Request (CR), "Revise OP-1014625 to Add Steam Condensate Clearing (U)" & $10 / 14 / 12$ \\
\hline CRF 2-1/2" Sintering Furnace Exam & $9 / 8 / 03$ \\
\hline LEU Liquid Blend Run Sheet, (J52L-16-02423 & $9 / 20 / 12$ \\
\hline $\begin{array}{l}\text { Qual test for Kernel Formation Process (AGR program) Employee: Monica } \\
\text { Culpepper; } \\
\text { OP-1014625 }\end{array}$ & $4 / 11 / 2012$ \\
\hline $\begin{array}{l}\text { Qual and Re-Qual for Wash / Dry For AGR Rev } 02 \text { (rev 06) Employee: Monica } \\
\text { Culpepper }\end{array}$ & $9 / 27 / 2012$ \\
\hline $\begin{array}{l}\text { OP-1015277 Operators Qualification Test Fluid Bed Sintering (WS145) for } \\
\text { Advanced Gas Reactor }\end{array}$ & taken $4 / 11 / 12$ \\
\hline Process Variable Specification Sheet (PVSS) for ADUN Blending, (J52L-16-02423) & $9 / 17 / 12$ \\
\hline $\begin{array}{l}\text { PVSS signature screen shot } \\
\text { PPO no. (tracking): } 000001107394 \\
\text { Parent serial no.: J52L-16-59516 }\end{array}$ & $12 / 18 / 12$ \\
\hline OP-0061161, Training of Uranium Processing Operators & 10 \\
\hline $\begin{array}{l}\text { PVSS signature screen shot } \\
\text { PPO no. (tracking): } 000001105253 \\
\text { Parent serial no.: J52L-16-29128 } \\
\end{array}$ & $1 / 24 / 13$ \\
\hline $\begin{array}{l}\text { PVSS signature screen shots } \\
\text { PPO no. (tracking): } 000001105247 \\
\text { Parent serial no.: J52L-16-29122 } \\
\end{array}$ & $12 / 5 / 12$ \\
\hline $\begin{array}{l}\text { Revision History Log, "Revise OP-1014625 to Add Steam Condensate Clearing } \\
\text { (U)," Change Review Minutes }\end{array}$ & $\begin{array}{l}10 / 8 / 12- \\
10 / 19 / 12\end{array}$ \\
\hline Solumina read and accept area training for G73AB-NU-59116 & NA \\
\hline N-50 "Employee Review of Area Documents (U)" & Revision 5 \\
\hline $\begin{array}{l}\text { Operator Qualification Test ADUN Solution Prep (Advanced Gas Reactor), OP- } \\
\text { 1014602; Revision } 18 \text { (Randy Steele) }\end{array}$ & $1 / 27 / 15$ \\
\hline $\begin{array}{l}\text { Operator Qualification Test ADUN Solution Prep (Advanced Gas Reactor), OP- } \\
\text { 1014602; Revision } 18 \text { (Monica Culpepper) }\end{array}$ & $10 / 20 / 14$ \\
\hline AGR 2.5in Sintering Furnace Data Collection: Batch G73AB-NU-59116 & NA \\
\hline
\end{tabular}




\section{Configuration Design and Control}

Procedures must be in place to implement configuration management of processes and equipment affecting quality. The impact on quality of changes to the configuration must be recognized before implementation, analyzed against the design bases/requirements, approved by responsible persons, and documented.

B\&W NOG-L controls equipment configuration via a change request generated in accordance with Quality Work Instruction QWI 5.1.12, "Change Management." Proposed changes to equipment and processes are reviewed by subject matter experts and management to ensure that no unforeseen adverse consequences may be created by the change. Once concurrences and unit manager approval are obtained, a work order is initiated to make the configuration change.

A recent occurrence involving the installation of a Monel valve in the ADUN system was not a result of inadequate configuration control. The replacement was specified to be a "like kind" replacement with a stainless steel valve. Further information on this incident can be found in Appendix 2.

Configuration design and control processes at B\&W NOG-L were found to be effective and compliant with NQA-1 requirements. Documents reviewed to assess the effectiveness of the B\&W NOGL configuration control practices (change management procedure and an example case) are referenced in Table 4.

Table 4. Objective evidence for configuration control of equipment and processes.

\begin{tabular}{|l|c|}
\hline \multicolumn{1}{|c|}{ Evidence Reviewed } & Revision or Date \\
\hline Quality Work Instruction 5.1.12, "Change Management" & 26 \\
\hline $\begin{array}{l}\text { CR-1042992 Change Request (CR), "Alteration to Power Guard on WS140 Power } \\
\text { Supply" }\end{array}$ & $7 / 30 / 14$ \\
\hline $\begin{array}{l}\text { CR-1042992 Revision History Log, "Alteration to Power Guard on WS140 Power } \\
\text { Supply" }\end{array}$ & NA \\
\hline $\begin{array}{l}\text { Form-N-51, Change Review Minutes, "Alteration to Power Guard on WS140 Power } \\
\text { Supply" }\end{array}$ & $7 / 13 / 14-8 / 4 / 14$ \\
\hline
\end{tabular}

\section{Operator Training, Certification, and Qualification}

Personnel performing work on activities affecting product quality are to be trained, certified, and qualified using a formal training program.

B\&W NOG-L requires operating personnel to pass written exams and to demonstrate proficiency on a process to be qualified or certified on a process. Operating personnel are made aware of revisions to operating instructions/procedures and are required to sign a training roster to document training on revisions.

All but one of the B\&W NOG-L operating personnel previously qualified on the kernel forming and sintering processes have since left the employ of B\&W NOG-L. The remaining experienced and previously qualified person will be used in the restart of the kernel forming and sintering processes and will assist in the on-the-job training of operating personnel during their initial qualification on the processes.

Operating personnel have completed written exams on the latest revisions to the procedures, but these exams had not been reviewed for external release and were not available to the review team at the time of review. Unclassified training records dated in 2012, prior to the previous kernel fabrication campaign, were presented as objective evidence during the review. Some deficiencies were noted in the 
documentation of the previous training (see Process Record Control and Accessibility). During the preparation of this report, B\&W NOG-L provided copies of recent qualification exams for two operators. Both exams were graded, signed and dated by the grading engineer, and signed and dated by a manager to authenticate the records. Training records reviewed by the team are shown in Table 5.

A continuing concern is the availability of qualified operating personnel. One of the operators undergoing training for the kernel forming and sintering processes has recently announced retirement. B\&W NOG-L management is continuing an effort to attain a full staffing contingent. One operator will be needed to replace the retiree to ensure uninterrupted operation of the kernel line.

$\mathrm{B} \& \mathrm{~W}$ NOG-L is ready in this area for line start-up. The issue identified requiring resolution prior to start-up of the kernel line (RI007) has been resolved.

Table 5. Objective evidence for operator training, certification, and qualification.

\begin{tabular}{|l|c|}
\hline \multicolumn{1}{|c|}{ Evidence Reviewed } & Revision or Date \\
\hline CRF 2-1/2" Sintering Furnace Exam & $9 / 8 / 03$ \\
\hline $\begin{array}{l}\text { Qual test for Kernel Formation Process (AGR program) Employee: Monica } \\
\text { Culpepper; } \\
\text { OP-1014625 }\end{array}$ & $4 / 11 / 2012$ \\
\hline $\begin{array}{l}\text { Qual and Re-Qual for Wash / Dry For AGR Rev 02 (rev 06) Employee: Monica } \\
\text { Culpepper }\end{array}$ & $9 / 27 / 2012$ \\
\hline $\begin{array}{l}\text { OP-1015277 Operators Qualification Test Fluid Bed Sintering (WS145) for } \\
\text { Advanced Gas Reactor }\end{array}$ & taken 4/11/12 \\
\hline Solumina read and accept area training for G73AB-NU-59116 & NA \\
\hline $\begin{array}{l}\text { Operator Qualification Test ADUN Solution Prep (Advanced Gas Reactor), OP- } \\
\text { 1014602; Revision 18 (Randy Steele) }\end{array}$ & $1 / 27 / 15$ \\
\hline $\begin{array}{l}\text { Operator Qualification Test ADUN Solution Prep (Advanced Gas Reactor), OP- } \\
1014602 ; \text { Revision 18 (Monica Culpepper) }\end{array}$ & $10 / 20 / 14$ \\
\hline
\end{tabular}

\section{Chemical Inventories}

Having an adequate chemical inventory (within the indicated useful chemical shelf-life and meeting chemical purity specifications) to start production and a plan to augment the inventory as necessary to complete production is not specified in the ASME NQA-1-2008, 1a-2009 standard, but it is a consideration for the AGR program.

$\mathrm{B} \& \mathrm{~W}$ NOG-L provided a listing of chemicals on-hand and presented the release forms authorizing their use in the AGR LEUCO kernel fabrication activities. At the close of the readiness review, B\&W NOG-L provided the chemical quantities on-hand, indicated "retest" dates, and how many forming runs each chemical could support. All liquid and dry chemicals with retest dates will not require retesting during the kernel fabrication interval. All liquid and dry chemicals, with exceptions of the carbon black slurry and Tween 20 surfactant, are sufficient to complete kernel fabrication. The carbon black slurry in inventory can support approximately eight forming runs, which will take months to complete, and procurement of an additional quantity of carbon black slurry has been authorized.

The carbon black slurry is an "off the shelf" item offered by the fabricator and no longer an experimental product. The additional quantity of carbon black slurry is expected to be received well in advance of the need date. Tween 20 is an inexpensive, off-the-shelf surfactant available through several vendors. B\&W NOG-L has identified a supplier of Tween 20 that also issues a certificate of analysis for 
impurities. Tween 20 will not be needed the first batch (or two) of kernels, but will be needed shortly thereafter to complete testing to reduce kernel fissure fractions.

Hydrogen inventory is presently adequate to start kernel sintering, although a requisition is already in place to replace the hydrogen trailer with a non-mobile tank installation. This work is expected to be completed before hydrogen is needed for kernel sintering.

The argon supply tank is frequently filled to ensure adequate supply to support the AGR and other fuel fabrication activities throughout the B\&W NOG-L facilities. The tank is refilled before it is depleted. The AGR program has not been interrupted by a lack of argon since AGR fuel fabrication started at B\&W NOG-L.

Ultra high purity (UHP) carbon monoxide inventories are more than adequate to complete the kernel fabrication activities, except it was noted that bottles received from an Airgas plant in New Jersey in 2012 lacked a retest date for the gas on the certificates of analysis whereas bottles received from an Airgas plant in Texas in 2010 indicated a retest date of 2013. At the time of the review, B\&W NOG-L was in communication with Airgas to understand the difference in certificates and whether the retest date on the Texan bottles is meaningful. Subsequent to the review, Airgas reported that the UHP carbon monoxide does not degrade with time and that the "retest date" on the certificate of analysis from the New Jersey gas plant was used to indicate the period of retention for the certificates of analysis and not a chemical shelf life.

B\&W NOG-L has sufficient chemicals in inventory to start the kernel forming and sintering processes. B\&W NOG-L is conditionally ready. The inventory will be sufficient to complete forming and sintering activities once additional carbon black slurry is received and Tween 20 surfactant is procured. A list of the documents reviewed to assess the adequacy of the chemical inventory is given in Table 6 .

Two issues were identified during the review that required resolution prior to start-up of the kernel line, RI005 and RI006. Both of these issues have been resolved at the time of the report and B\&W NOG$\mathrm{L}$ is deemed ready in this area for kernel line start-up.

Table 6. Objective evidence for chemical inventory and suitability.

\begin{tabular}{|l|c|}
\hline \multicolumn{1}{|c|}{ Evidence Reviewed } & Revision or Date \\
\hline $\begin{array}{l}\text { Air Products Certificate of Analysis } \\
\text { Hydrogen Unit 816214 ticket \#5183B95950 }\end{array}$ & $11 / 30 / 12$ \\
\hline $\begin{array}{l}\text { Airgas Certificate of Batch Analysis } \\
\text { Lot 82-124303058-1, UHP carbon monoxide }\end{array}$ & $2 / 13 / 12$ \\
\hline $\begin{array}{l}\text { Airgas Certificate of Batch Analysis } \\
\text { Lot 49-124195288-1, UHP carbon monoxide (WAE358, WAE354, WAE353) }\end{array}$ & $1 / 29 / 10$ \\
\hline $\begin{array}{l}\text { Airgas Certificate of Batch Analysis } \\
\text { Lot 49-124195288-1, UHP carbon monoxide (WAE356, WAE355, WAE359) }\end{array}$ & $1 / 29 / 10$ \\
\hline $\begin{array}{l}\text { Airgas Certificate of Batch Analysis } \\
\text { Lot 49-124209785-1, UHP carbon monoxide (WAE357) }\end{array}$ & $2 / 18 / 10$ \\
\hline Chemical inventory calculations (spreadsheet) & $\mathrm{NA}$ \\
\hline Chemical inventory list (doc) & $\mathrm{NA}$ \\
\hline Form M35-037 (unclassified extract - reorder of process gases) & NA \\
\hline Inventory of graphite sintering tubes & NA \\
\hline PA/SQC Bulk/Cylinder Gas Release CO-21 (carbon monoxide) & $5 / 25 / 10$ \\
\hline
\end{tabular}




\begin{tabular}{|l|c|}
\hline \multicolumn{1}{|c|}{ Evidence Reviewed } & Revision or Date \\
\hline PA/SQC Bulk/Cylinder Gas Release CO-24 (carbon monoxide) & $2 / 23 / 12$ \\
\hline PA/SQC Bulk/Cylinder Gas Release CO-27 (carbon monoxide) & $12 / 11 / 12$ \\
\hline PA/SQC Bulk/Cylinder Gas Release H-009 (hydrogen) 5183B95950 & $12 / 4 / 12$ \\
\hline $\begin{array}{l}\text { Quality Engineering Release For INL Contract Consumables, Materials, and Product, } \\
\text { MISC.-62 (multiple chemicals) }\end{array}$ & $9 / 12 / 12$ \\
\hline $\begin{array}{l}\text { Quality Engineering Release For INL Contract Consumables, Materials, and Product, } \\
\text { MISC.-63 (urea, nitric acid, hexamethylenetetramine) }\end{array}$ & $9 / 12 / 12$ \\
\hline $\begin{array}{l}\text { Quality Engineering Release For INL Contract Consumables, Materials, and Product, } \\
\text { MISC.-64 }\end{array}$ & $11 / 19 / 12$ \\
\hline $\begin{array}{l}\text { Quality Engineering Release For INL Contract Consumables, Materials, and Product, } \\
\text { MISC.-65 (urea) }\end{array}$ & $12 / 12 / 12$ \\
\hline $\begin{array}{l}\text { Quality Engineering Release For INL Contract Consumables, Materials, and Product, } \\
\text { MISC.-66 (trichloroethylene) }\end{array}$ & $1 / 21 / 13$ \\
\hline $\begin{array}{l}\text { Quality Engineering Release For INL Contract Consumables, Materials, and Product, } \\
\text { MISC.-67 (nitric acid, ammonium hydroxide) }\end{array}$ & $8 / 20 / 14$ \\
\hline $\begin{array}{l}\text { Quality Engineering Release For INL Contract Consumables, Materials, and Product, } \\
\text { MISC.-71(hexamethylenetetramine) }\end{array}$ & $1 / 14 / 15$ \\
\hline SFF Gas Inventory for Sintering (hydrogen and carbon monoxide) & NA \\
\hline $\begin{array}{l}\text { Email: Will Westgate, Account Manager, Airgas USA, LLC to Joseph Keeley (B\&W } \\
\text { NOG-L) regarding the UHP carbon monoxide retest date. }\end{array}$ & $1 / 15 / 15$ \\
\hline
\end{tabular}

\section{Material Procurements}

Material procurement processes and records of purchases (except as noted above for chemical inventory) were not included in the review. Material procurements have passed previous scrutiny during supplier qualification audits.

\section{Operability of Essential Kernel Fabrication Equipment}

A walk-down of the processing floor was conducted to visually inspect cleanliness, evidence of deterioration, currency of instrument calibrations, and unresolved maintenance issues.

The kernel forming and wash/dry stations were inspected. No evidence of deterioration was observed and the equipment appeared to be in good repair and clean. Every instrument relied upon for quality had a current calibration. B\&W NOG-L self-identified that the wash/dry station configuration was inaccurately reflected in the nuclear criticality safety (NCS) models and had taken action to correct the models prior to the readiness review. The model revision is in progress. While awaiting the outcome of the revised model, the wash/dry station is out-of-service.

The $2 \frac{1}{2}$ " sintering furnace is being readied for use. Updates to the Facility Alarm System (FAS) and verification of Items Relied on for Safety (IROFS) are in progress. The $2 \frac{1}{2}$ " furnace is needed to test changes to the kernel wash, dry, and calcining procedures for effectiveness in reducing the kernel fissure fraction. The $2 \frac{1}{2}$ " sintering furnace is out-of-service until the FAS and IROFS updates and verifications are completed. Whereas the $2 \frac{1}{2}$ " furnace has not been operated in five years, the system will need to 
undergo vacuum-leak checks and heating element/insulation bake-out procedures before it will be operable. B\&W NOG-L provided a schedule for these activities.

The 6" sintering furnace, which is needed for calcining and sintering the AGR-5/6/7 kernels, is outof-service. This furnace will not be needed until testing with the $2 \frac{1}{2}$ " sintering furnace is completed and the product analyzed. Priority is given to restoring the $2 \frac{1}{2}$ " sintering furnace functionality over that of the 6 " sintering furnace. The 6" sintering furnace is not needed to start kernel forming activities. B\&W NOGL has provided a checklist of work activities necessary to restore the 6" furnace to an operable status, but no schedule is available. Nonetheless, action items on the checklist are in progress. Expectations are that the $2 \frac{1}{2}$ " and 6"furnaces will be ready for operation before each one is needed. The review team grants $\mathrm{B} \& \mathrm{~W}$ NOG-L a conditional readiness status for this criterion.

This area was found to be NQA-1 compliant however; identified issues were identified that require resolution prior to line start-up. They are RI001, RI002, RI003, and RI004.

Table 7. Objective Evidence for Essential Kernel fabrication Equipment

\begin{tabular}{|l|c|}
\hline \multicolumn{1}{|c|}{ Evidence Reviewed } & Revision or Date \\
\hline Thermocouple, CRFT-00307, Type C, 24 inch & $11 / 20 / 2014$ \\
\hline N-74, "AGR Forming Proof of Readiness" & $2 / 3 / 15$ \\
\hline
\end{tabular}

\section{Corrective Action System Effectiveness to Capture "Lessons Learned"}

As conditions adverse to quality are identified, a corrective action process is needed to ensure that the condition is corrected within a reasonable time period, that the significance of the consequences from the condition is assessed, that causes for the significant condition are identified, and that actions are taken to prevent recurrence. The investigation and categorization of "significant" conditions adverse to quality need to be documented.

B\&W NOG-L has a corrective action process determined to be compliant with the NQA-1 requirements during a supplier qualification audit jointly conducted by INL and Pacific Northwest National Laboratory (PNNL). B\&W NOG-L has a 3-tiered approach for corrective actions. Level 1 conditions deemed "significant" ( QWI 14.1.1 Attach 1, Preventive / Corrective Action System Severity Levels) as given in NQA-1 and these are formally investigated to determine causal factors, corrective actions are determined (QWI 14.1.1 Attach 6, Incident Investigations / Error Precursor Analaysis), and senior staff is apprised of the investigation and the status of corrective actions (QWI 14.1.1 Attach 9, Roadmap for Response to Significant Events). The investigation and corrective actions are documented and tracked to completion.(QWI 14.1.1 Attach 3, Preventive / Correct Action System Process Flow Diagram) Conditions that may impact other programs are communicated plant-wide.

Level 2 incidents are investigated by convening a critique with a facilitator, frontline management, and knowledgeable and involved personnel. Corrective actions are defined and senior staff is apprised of the status. A formal root-cause investigation is not mandated.

Level 3 incidents are minor and insignificant in nature and are corrected without requiring that a critique be convened.

A recent incident involving the inadvertent installation of a Monel 3-way valve in the place of a stainless steel 3-way valve on the ADUN dissolvers was deemed to be a Level 2 incident (CA 20141998). A critique of the event was held to determine the time line of events and causal factors into the event. Corrective actions were taken; namely, 
- ADUN processing was halted

- ADUN samples submitted for chemical analysis

- Components recently changed out were inspected and the Monel valve was located

- Full inspection of the ADUN system was conducted to ensure no other components were fabricated from incompatible materials

- A 400-series mixing blade was replaced with a 300-series blade because the 400 -series blade showed some evidence of etching

- Monel valves and fittings have been segregated from stainless steel components

At the time of the readiness review, B\&W NOG-L was conducting a formal taproot investigation (meeting Department of Energy requirements) of the incident because of the delays to the processing schedule and attention received from customers. The review team did not request any in-process documentation of the taproot investigation so as to avoid interfering with the investigation or the independence of the conclusions that will be forthcoming. More detail of the event can be found in Appendix 2.

\section{Product Acceptance}

Product acceptance processes were captured in a variety of procedures with acceptance criteria. These documents are listed in Table 8.

Acceptance of a product is determined as an end-result of the chemical and/or physical analyses and adherence to limited process parameter specifications. In process indicators of time, temperature and pressure were used to give confidence that the systems are working. Final acceptance was made by comparing the analytical results to INL specification after applying the statistical confidence intervals. The review identified how these procedures were used and how the material was delivered from one process to the following process until the final results were obtained.

Once material was delivered to the Chemical Analysis group, it was handled in a clean room environment to ensure accuracy of the results. Analyses are performed with sample blanks and concentration standards traceable to NIST chemical standards. These processes are in place and ready for use. No additional preparations were needed.

Table 8. Objective Evidence for Product Acceptance

\begin{tabular}{|l|c|}
\hline \multicolumn{1}{|c|}{ Evidence Reviewed } & $\begin{array}{c}\text { Revision or } \\
\text { Date }\end{array}$ \\
\hline $\begin{array}{l}\text { Information management System Aspen or Sample Master } \\
\text { (data transferred to electronic media from the Data Cards); }\end{array}$ & $1 / 14 / 2015$ \\
\hline OP-1000312, "Tamper Safe Sealing Products" & rev 20 \\
\hline $\begin{array}{l}\text { OP-1004132, "Impurity Analyses in Uranium Oxide/Carbide and Metal Material by ICP- } \\
\text { MS" }\end{array}$ & rev 1 \\
\hline OP-1004139, "Uranium Isotropic Analyses in Uranium Based Material by ICP-MS" & rev 1 \\
\hline OP-1009921, "Process Monitor in SFF" & rev 5 \\
\hline OP-1014613 "Washing /Drying Operations Advanced Gas Reactor Program" & rev 3 \\
\hline $\begin{array}{l}\text { OP-1020491, "Sampling of Various Material Types for Material Control and } \\
\text { Accountability" " }\end{array}$ & rev 04 \\
\hline OP-1022961, "Sintering for AGR and Other SFF Contracts in 2.5 Inch Furnace at WS 140" & rev 4 \\
\hline PPO-0910001-004, "Preparation of UO3-C Kernels for AGR and Other" & rev 4 \\
\hline
\end{tabular}




\begin{tabular}{|l|c|}
\hline \multicolumn{1}{|c|}{ Evidence Reviewed } & $\begin{array}{c}\text { Revision or } \\
\text { Date }\end{array}$ \\
\hline Q2 179 Balance Check Book & rev 4 \\
\hline Q2-139 Data Card & rev 3 \\
\hline SPC-1363, “AGR-5/6 Fuel Fabrication Feedstock Chemical Purity Specification” & rev 2 \\
\hline
\end{tabular}

\section{Handling and Storage}

While no material was available to identify how the handling and storage processes were performed, we were given procedures and descriptions. Some of the processes reviewed included:

Table 9. Objective Evidence for Handling and Storage

\begin{tabular}{|l|c|}
\hline \multicolumn{1}{|c|}{ Evidence Reviewed } & $\begin{array}{c}\text { Revision or } \\
\text { Date }\end{array}$ \\
\hline OP-1000312, "Tamper Safe Sealing Products" & rev 20 \\
\hline $\begin{array}{l}\text { OP-1020491, "Sampling of Various Material Types for Material Control and } \\
\text { Accountability" }\end{array}$ & rev 04 \\
\hline
\end{tabular}

\section{Follow-up from Previous Reviews}

A readiness review and visits held at B\&W NOG-L facilities in Nov, 2013 identified four areas of concern that B\&W NOG-L addressed. Each of the following areas was briefly revisited to assess the effectiveness of the actions taken to address the concerns.

1. The weight set used to verify scale acceptance for use is not listed on the form created to document the scale acceptance. Traceability to NIST is lost without listing the weight set by ID number. (reference OP-1008157, Calibration and Standard Checks for Mass Measurement)

2. Training records were not authenticated by a signature of management and dated.

3. Procedures governing internal audits require auditors to make a statement regarding program/process effectiveness, but recent audits did not contain such a statement.

4. Resinated-graphite matrix powder has a not to exceed temperature limit of $35^{\circ} \mathrm{C}$. No measuring device is monitoring the temperature to determine whether or not the material temperature is exceeded.

Evidence was identified that each of these areas had been addressed in one form or another, however, the records authentication continued to show evidence of lack of completeness. This resulted in an issue requiring resolution (refer to section Process Record Control and Accessibility). Documents provided by B\&W NOG-L to INL during the preparation of this report are evidence that this concern has already been addressed. Training records include a signature block for the frontline manager to sign and date to authenticate the record.

Table 10. Objective Evidence for satisfactory resolution of concerns from a previous review.

\begin{tabular}{|c|c|}
\hline Evidence Reviewed & $\begin{array}{c}\text { Revision or } \\
\text { Date }\end{array}$ \\
\hline N-50 "Employee Review of Area Documents (U)" & Revision 5 \\
\hline
\end{tabular}


Operator Qualification Test ADUN Solution Prep (Advanced Gas Reactor), OP1014602; Revision 18 (Randy Steele)

$1 / 27 / 15$

Operator Qualification Test ADUN Solution Prep (Advanced Gas Reactor), OP1014602; Revision 18 (Monica Culpepper)

$10 / 20 / 14$ 


\section{Issues}

The information provided below indicates a potential deficiency. Please provide any objective evidence that could alleviate or eliminate the deficiency. If no objective evidence is provided by the end of the scheduled fieldwork, the issue identified below will be included in the audit report and reported as a deficiency.

Audit Title: Kernel Fabrication Readiness Review

Auditor Name: Kirk Bailey / Doug Marshal

Potential Deficiency Description:

The $2 \frac{1}{2}$ " furnace (WS-140) is out-of-service pending facility alarm system (FAS) and items relied on for safety (IROFS) review and release.

Requirement (PRD and Procedure references): B\&W NOG-L internal process requirement.

Discussion (include "Contrary to" statement):

Recommended Corrective Action: B\&W NOG-L completes the FAS and IROFS verifications.
Audit No.: IAS15921 RI001

Date Problem Found: 1/15/2015

\section{Discussion (include "Contrary to" statement):}


The information provided below indicates a potential deficiency. Please provide any objective evidence that could alleviate or eliminate the deficiency. If no objective evidence is provided by the end of the scheduled fieldwork, the issue identified below will be included in the audit report and reported as a deficiency.

Audit Title: Kernel Fabrication Readiness Review

Audit No.: IAS15921 RI002

Auditor Name: Kirk Bailey / Doug Marshal

Date Problem Found: 1/15/2015

Potential Deficiency Description:

The 6" furnace (WS-145) is out-of-service and not ready for operation. The status of WS-145 does not need to be changed prior to operating either the forming columns or WS-140.

Requirement (PRD and Procedure references): B\&W NOG-L internal requirements.

Discussion (include "Contrary to" statement):

Recommended Corrective Action: B\&W NOG-L completes the checklist of actions needed to restore equipment operability, including instrument calibrations and verification of any outstanding IROFS. 
The information provided below indicates a potential deficiency. Please provide any objective evidence that could alleviate or eliminate the deficiency. If no objective evidence is provided by the end of the scheduled fieldwork, the issue identified below will be included in the audit report and reported as a deficiency.

Audit Title: Kernel Fabrication Readiness Review

Audit No.: IAS15921 RI003

Auditor Name: Kirk Bailey / Doug Marshal

Date Problem Found: 1/15/2015

Potential Deficiency Description:

The kernel gel wash and dry stations are out-of-service because the nuclear criticality safety Monte Carlo model used for criticality safety calculations does not reflect the current as-built configuration inside the hood. Notably, this was self-identified by B\&W NOG-L and resolution was in progress before the readiness review was conducted. The reason for the discrepancy is a B\&W NOG-L concern.

Requirement (PRD and Procedure references): B\&W NOG-L internal procedure requirement

Discussion (include "Contrary to" statement):

Recommended Corrective Action: B\&W NOG-L completes update of nuclear criticality safety documents and modelling of current equipment configuraiton. 
The information provided below indicates a potential deficiency. Please provide any objective evidence that could alleviate or eliminate the deficiency. If no objective evidence is provided by the end of the scheduled fieldwork, the issue identified below will be included in the audit report and reported as a deficiency.

Audit Title: Kernel Fabrication Readiness Review

Auditor Name: Kirk Bailey/ Doug Marshal
Audit No.: IAS15921 RI004

Date Problem Found: 1/15/2015

Potential Deficiency Description:

Forming column solution pumps and their settings are determined by adjusting the pump rate and measuring the mass of pumped fluid over a 3 minute time interval. The calculated flow rate and mass flow meter readings need to be compared and logged. This practice enables the uncalibrated flow meter readings to be used as relative measures for solution flow rates, thus allowing the meter readings to be used in verifying that flow rates remain in the $\pm 5 \%$ window given in the operating procedure.

Requirement (PRD and Procedure references):

NQA-1-2008/1a-2009 requirement 11, paragraph 300 Test Procedures: Test procedures shall include or reference the test configuration and test objectives. Test procedures shall also include provisions for assuring that prerequisites and suitable environmental conditions are met, adequate instrumentation is available and used, appropriate tests and equipment are used, and necessary monitoring is performed.

Discussion (include "Contrary to" statement):

Recommended Corrective Action: B\&W NOG-L to establish a log book to record flow meter readings when measuring flow rates to establish a correspondence between the uncalibrated flow meter reading and measured flow rates for reference by operating personnel during the kernel forming runs. 
The information provided below indicates a potential deficiency. Please provide any objective evidence that could alleviate or eliminate the deficiency. If no objective evidence is provided by the end of the scheduled fieldwork, the issue identified below will be included in the audit report and reported as a deficiency.

Audit Title: Kernel Fabrication Readiness Review

Audit No.: IAS15921 RI005

Auditor Name: Kirk Bailey/Doug Marshal

Date Problem Found: 1/15/2015

Potential Deficiency Description:

- Airgas Certificates of Batch Analysis for three UHP-carbon monoxide gas lots were presented as objective evidence of readiness. Two of the gas batches were analyzed by a Texas laboratory in 2010 , which have been lapsed "retest" date since 2013. The third gas batch was analyzed by a New Jersey laboratory in 2012 , but does not include a retest date. Whether the UHP-CO is suitable for use is indeterminate until the meaning of the retest dates on two batches and the lack of same on the other are understood and addressed.

Requirement (PRD and Procedure references):

NQA-1-2008/1a-2009; Requirement 7, para 503:

When a Certificate of Conformance is used, the minimum criteria of paras. 503(a) through (f) of this Requirement shall be met.

(b) The certificate shall identify the specific procurement requirements met by the purchased material or equipment, such as codes, standards, and other specifications.

Discussion (include "Contrary to" statement):

Recommended Corrective Action: B\&W NOG-L contacted Airgas to clarify the meaning of the retest date and the absence of a retest date on some of the cylinders of ultra-high purity carbon monoxide (UHP-CO). Airgas indicated that the practice at the Texas plant is to use the retest date to indicate the period of record retention for the certificate of analysis and not an expiration date for the UHP-CO. The New Jersey plant does not use the retest date for their records retention. Airgas confirmed that the UHP-CO does not have an expiration date. The issue is resolved. 
The information provided below indicates a potential deficiency. Please provide any objective evidence that could alleviate or eliminate the deficiency. If no objective evidence is provided by the end of the scheduled fieldwork, the issue identified below will be included in the audit report and reported as a deficiency.

Audit Title: Kernel Fabrication Readiness Review

Audit No.: IAS15921 RI006

Auditor Name: Kirk Bailey / Doug Marshal

Date Problem Found: 1/15/2015

Potential Deficiency Description:

- The solid and liquid chemical inventory needs to be compared with what is needed to start and to complete kernel fabrication. Any shortfall in the inventory requires objective evidence that the needed chemicals will be acquired in time for processing (e.g., minimum inventory triggers for reordering, purchase orders, or procurement plan).

Requirement (PRD and Procedure references): None. INL asked for evidence that sufficient chemicals will be on-hand to start and to finish fuel kernel fabrication, that chemicals are within any reported shelf-life, and that a plan is in place to acquire any needed chemicals.

Discussion (include "Contrary to" statement):

Recommended Corrective Action: B\&W NOG-L has provided a spreadsheet linking current chemical inventory with the program needs for fuel kernel fabrication. Some concerns were noted; namely, certificates of analysis for some ultra-high purity carbon monoxide gas cylinders indicated a lapsed retest date (see IAS15921 RI005 for details), and inventories for carbon black slurry and Tween 20 surfactant were insufficient for the planned kernel fabrication. B\&W NOG-L has submitted a request to acquire more carbon black and identified a ready source of surfactant that comes with an impurity analysis. 
The information provided below indicates a potential deficiency. Please provide any objective evidence that could alleviate or eliminate the deficiency. If no objective evidence is provided by the end of the scheduled fieldwork, the issue identified below will be included in the audit report and reported as a deficiency.

Audit Title: Kernel Fabrication Readiness Review

Audit No.: IAS15921 RI007

Auditor Name: Kirk Bailey / Doug Marshal

Date Problem Found: 1/15/2015

Potential Deficiency Description:

- A concern was noted when reviewing old operator training records for kernel fabrication in 2012. Training exams were presented as objective evidence that lacked signatures from the cognizant engineer or front-line manager, dates, procedure revision numbers, and evidence that the exams were graded. This is noted as a weakness only because the operator qualifications have expired and will need to be redone.

Requirement (PRD and Procedure references):

NQA-1-2008/1a-2009; requirement 17; para 300 Authentication of Records

Documents shall be considered valid records only if stamped, initialed, or signed and dated by authorized personnel or otherwise authenticated.

Discussion (include "Contrary to" statement):

Recommended Corrective Action: B\&W NOG-L has resolved the issue and provided evidence that recent exams have been properly graded and authenticated. Training records have been revised to include blocks for front-line manager signature and date. 
The information provided below indicates a potential deficiency. Please provide any objective evidence that could alleviate or eliminate the deficiency. If no objective evidence is provided by the end of the scheduled fieldwork, the issue identified below will be included in the audit report and reported as a deficiency.

Audit Title: Kernel Fabrication Readiness Review

Audit No.: IAS15921 UI001

Auditor Name: Kirk Bailey / Doug Marshal

Potential Deficiency Description:

- Some QA records, specifically Form N-50, lack the authenticating manager signature and date as required in NQA-1 Requirement 17300 (a). This concern was noted in a previous readiness review, but has not been adequately resolved or implemented.

Requirement (PRD and Procedure references):

NQA-1-2008/1a-2009; requirement 17; para 300 Authentication of Records

Documents shall be considered valid records only if stamped, initialed, or signed and dated by authorized personnel or otherwise authenticated.

Discussion (include "Contrary to" statement):

Recommended Corrective Action: B\&W NOG-L has resolved this issue. Form N-50 and training record forms have been revised to include line-management signature and date to authenticate records. 
The information provided below indicates a potential deficiency. Please provide any objective evidence that could alleviate or eliminate the deficiency. If no objective evidence is provided by the end of the scheduled fieldwork, the issue identified below will be included in the audit report and reported as a deficiency.

Audit Title: Kernel Fabrication Readiness Review

Audit No.: IAS15921 UI002

Auditor Name: Kirk Bailey / Doug Marshal

Date Problem Found: 1/15/2015

Potential Deficiency Description:

- Instrument calibrations appear to be in order, but a weakness was noted. The nature of work performed at B\&W NOG-L does not lend itself well to the use of external agencies to certify the calibration services laboratory to nationally recognized performance standards, such as ISO 10012 or ISO 17025 . Any link between the calibration services and the performance standards could not be verified and remains indeterminate.

Requirement (PRD and Procedure references):

NQA-1-2008/1a-2009; requirement 12; para 303 Control

Calibration procedures shall identify or reference required accuracy and shall define methods and frequency of checking accuracy. The calibration method and interval of calibration shall be based on the type of equipment, stability characteristics, required accuracy, intended use, and other conditions affecting performance.

Discussion (include "Contrary to" statement):

This is a precautionary concern as the condition stated was that the calibration processes could not be fully reviewed.

NOTE: all records reviewed were properly documented and equipment in service is within calibration dates.

Recommended Corrective Action:

B\&W NOG-L to ensure calibration procedures and records are compliant to NQA-1 requirements and can demonstrate this relationship through documents and records. 
The information provided below indicates a potential deficiency. Please provide any objective evidence that could alleviate or eliminate the deficiency. If no objective evidence is provided by the end of the scheduled fieldwork, the issue identified below will be included in the audit report and reported as a deficiency.

Audit Title: Kernel Fabrication Readiness Review

Audit No.: IAS15921 UI003

Auditor Name: Kirk Bailey / Doug Marshal

Date Problem Found: 1/15/2015

Potential Deficiency Description:

- No objective evidence was presented regarding the "periodic" evaluation of technician skills for the calibration services that they provide.

Requirement (PRD and Procedure references):

NQA-1-2008/1a-2009; requirement 2; para 302 Inspection and Test

The initial capabilities of a candidate shall be determined by an evaluation of the candidate's education, experience, training, and either test results or capability demonstration. The job performance of inspection and test personnel shall be reevaluated at periodic intervals not to exceed 3 years.

Discussion (include "Contrary to" statement):

Contrary to the requirement to provide periodic review of a candidate's ability to perform, no objective evidence was found to support periodic review. No documented practice established a requirement that a candidate job performance should be reviewed within a period not to exceed 3 years.

Recommended Corrective Action:

Include a clear description of the management of Inspection and Test personnel that states how a candidate's job performance is reviewed. 


\section{Appendix 1}

\section{Readiness Review Criteria AGR-5/6/7 LEUCO Kernel Fabrication}

The following focus areas are subject to scrutiny by the readiness review team as each applies to LEUCO kernel fabrication activities (e.g., forming, washing, drying, calcining, sintering, upgrading, etc.). An effort has been made by INL to be complete in the focus areas, but the reviewers will respond to the information and objective evidence obtained and follow leads as deemed necessary. A visit to the processing area is requested to allow reviewers to see the equipment and make limited inspections of calibration stickers, equipment condition, etc.

References to NQA-1 mean ASME-NQA-1-2008/1a-2009. Even though the outline below does not quote the entire text of the paragraph within a NQA-1 requirement, objective evidence may be requested by the review team to demonstrate compliance with all requirements within the referenced paragraph.

I. Process instrumentation and M\&TE calibrations

a. Tools, gages, instruments, etc. are controlled, calibrated, adjusted, and maintained to required accuracy limits (NQA-1 Req't 12 ๆ 100)

b. Calibrations performed at prescribed intervals against traceable or certified equipment/standards (NQA-1 Req't 12 ๆ 301)

c. M\&TE consistently found out of calibration is repaired or replaced (NQA-1 Req't 12 ๆ 303)

d. When M\&TE is found out of calibration or damaged, data collected since the last known acceptable calibration shall be evaluated for acceptability (NQA-1 Req't 12 १ 303.2)

e. M\&TE calibration status can be readily determined by inspection and documented (NQA-1 Req't 12 ๆ 303.6, 401 - 402)

II. Approval, control, and accessibility of operating procedures

a. Activities affecting quality prescribed by and performed in accordance with documented instructions, procedures, etc. (NQA-1 Req't 5 ๆ 100)

b. Operating procedures and instructions are controlled to ensure the latest revision is used (NQA-1 Req't 6 ๆ 100)

c. Operating procedures and instructions are reviewed and approved prior to releasing them for use (NQA-1 Req't 6 ๆ 100)

d. Documents and changes to documents are controlled (NQA-1 Req't 6 ๆ 200)

e. Test (process) requirements and acceptance criteria based upon documented specifications [NQA-1 Req't 11 ๆ 200(b); 300(a-b)]

f. Review, authorization, and control of operator instructions (e.g., recipe sheets)

III. Process record control and accessibility

a. Records are validated or authenticated by authorized personnel [NQA-1 Req't 17 १ 300(a)]

IV. Configuration design and control

a. Procedures established to implement configuration management and responsibilities/authorities are identified (NQA-1 Req't 3 ๆ 601)

b. Configuration changes affecting quality are (NQA-1 Req't 3 ๆ 601.1-601.9):

i. Recognized before implementation 
ii. Analyzed against design bases and requirements

iii. Approved by responsible persons

iv. Documented (incl. basis)

c. Controls are established to ensure that only correct and accepted items (e.g., materials of construction) are used or installed (NQA-1 Req't 8 ๆ 100 - 303)

V. Operator training, certification and qualification

a. Indoctrination and training (NQA-1 Req't 2 ๆ 200)

b. Performers indoctrinated and trained (NQA-1 Req't 2 ๆ 201)

c. Formal training program (NQA-1 Req't 2 ๆ 202)

VI. Chemical inventories

a. Chemical inventories are sufficient for expected needs

i. Traditionally used materials

ii. Specialty materials (e.g., Tween 20 surfactant) needed for testing

b. Chemical shelf-lives will not be exceeded during the period of kernel fabrication (NQA-1 Req't 8 ๆ 302)

c. Chemicals comply with SPC-1363, "AGR-5/6/7 Fuel Fabrication Feedstock Chemical Purity Specifications," Rev. 2

d. Miscellaneous consumables (e.g., graphite furnace parts, TCE, etc.) are adequate for expected needs

VII. Material procurements

a. Procured from qualified suppliers (or with commercial grade dedication) (NQA-1 Req't 4 q 100; NQA-1 Req't 7 ๆ 100, 700).

b. Technical (functional) requirements and non-conformance reporting requirements are communicated to suppliers (NQA-1 Req't 4 ๆ 202, 206; NQA-1 Req't 7 9 501 - 503)

c. Receiving inspection is performed to verify conformance with technical and functional requirements (NQA-1 Req't 7 9 505)

VIII. Operability of essential kernel fabrication equipment

a. Equipment walk-down has been performed to inspect equipment and instrumentation for:
i. Cleanliness
v. Current calibrations
ii. Deterioration or defects
vi. Plant utility availability
iii. Damaged or missing
vii. Sensor and alarm equipment functionality
iv. Maintenance issues
viii. Material compatibility

IX. Corrective action system effectiveness to capture "lessons learned"

a. Conditions adverse to quality identified and corrected ASAP (NQA-1 Req't 16 ๆ 100)

b. Significant conditions adverse to quality investigated to determine the cause and actions taken to prevent recurrence (NQA-1 Req't 16 \ 100)

c. Significant conditions and corrective actions are documented and reported to appropriate management (NQA-1 Req't 16 ๆ 100)

d. Completion of corrective actions is verified (NQA-1 Req't 16 ๆ 100)

X. Product acceptance

a. Inspection for acceptance by an independent, qualified person (NQA-1 Req't 10 १ 100) 
b. Inspection requirements and acceptance criteria are specified and documented (NQA-1 Req't 10 १ 200)

c. Sampling procedures based on standard statistical methods with engineering approval (NQA-1 Req't 10 ๆ 402) [INL sampling plan, PLN-4352 Rev. 3 is used]

d. Acceptance is approved by authorized personnel (NQA-1 Req't 10 ๆ 604)

e. Role of the analytical laboratory in this product acceptance

i. ASTM or other standards used

ii. Internal standards

XI. Handling and Storage

a. Product stored to prevent loss, damage, and minimize deterioration (NQA-1 Req't 13 ๆ 100, 200, 300)

b. Product containers are marked or labeled with special handling/storage requirements (NQA1 Req't 13 ๆ 600)

c. Controls are in place to prevent inadvertent use of non-conforming product [NQA-1 Req't 15 ๆ 100, 300(a-b)]

d. Non-conforming product shall be evaluated for alternative use or disposition (NQA-1 Req't 15 ๆ 401)

e. The disposition of non-conforming product shall be documented (NQA-1 Req't 15 q 404)

XII. Follow-up from previous reviews

a. Ensure corrective actions have been effective

i. Check weight ID's are recorded when used to verify balance functionality

ii. Training records are signed by management

iii. Internal audits reflect assessments of process effectiveness (if an audit has been performed for unclassified systems associated with AGR fuel fabrication) 


\section{Appendix 2}

\section{Corrective Action CA 20141998; Alloy 400 Valve}

B\&W NOG-L notified INL of a production run of ADUN material that yielded contaminated material. Batch J52R-93-01420 for the AGR Program was identified as having abnormal coloration. The batch was segregated and analyzed. Results from the analysis concluded that there was an unexpectedly high Nickel content and the amounts were unacceptable. The source of the nickel content was traced to a Alloy 400 (monel) valve that had been installed in the ADUN system during a recent maintenance order. The ADUN system is of stainless steel (SS) construction to minimize system contamination of the processed materials. The Alloy 400 valve was installed in error. It was removed and replaced with the appropriate SS valve. Some components of the system were replaced as they could not be completely cleared of the contaminant and the system was flushed and cleared of contaminant. A following run was processed and analyzed to verify acceptable system performance.

The following is a time line of events associated with this corrective action. Due to customer interest this issue was raised to a higher severity level requiring a tap root analysis by management request:

DATE

11/18/14 Abnormal coloration noted in batch

- Analysis determined high Ni content

- Material cannot be blended to reduce Ni levels

12/2/14 Level 2 CA 201401998 initiated per QWI 14.1.1

- Determined source of chemical contamination

- Alloy 400 (Monel) valve introduced into system during preparations for operation $(10 / 2 / 14)$

\section{Immediate Actions}

11/20/14 Work Stoppage

11/25/14 Customer notified

11/18/14 Segregated processed material

12/2/14 Visual Verification of remaining components in ADUN system for absence of incompatible material(s)

12/2/14 Notified Industrial Engineering of incident

12/3/14 Valve Replaced

$12 / 3 / 14$

$12 / 3 / 14$ thru

$12 / 11 / 14$

$12 / 2014$
Replaced pump to eliminate residual contaminate material

ADUN system flushing performed.

Verified that residual contaminates in flush were below trace level.

Discussed incident with operators and engineers

Increases awareness of visual similarity between SS and Alloy 400

Separation of stored Alloy 400 from SS parts - ongoing 
12/3/14 Critique Held -

- Release of report pending meeting with senior staff

- Tap Root Investigation (formal Causal Analysis) requested by management Investigation currently ongoing

\section{Future Events}

Causal factors being reviewed by management

Long Term Corrective Actions being determined

Systemic nature of event to be determined by investigation 